\title{
Dynamic Range Enhancement in Analog Optical Links with a Balanced Modulation and Detection Scheme
}

\author{
D.A.I. Marpaung, C.G.H. Roeloffzen and W. van Etten \\ University of Twente, Faculty of Electrical Engineering, Mathematics and Computer Science, \\ Telecommunication Engineering group, P.O. Box 217, 7500 AE, Enschede, The Netherlands
}

\begin{abstract}
A novel noise reduction scheme called Balanced Modulation and Detection (BMD) is proposed. In this scheme, the modulating RF signal is half-wave rectified in the optical domain, eliminating the DC optical power resulting from pre-biasing of the optical source. A link model employing this scheme has been developed and key parameters describing link performance have been calculated. A comparison with an externally-modulated link called the class-AB (CAB) photonic link is carried out. Improvement in link SNR and dynamic range up to $8.7 \mathrm{~dB}$ and $18 \mathrm{~dB}$, respectively, are obtained.
\end{abstract}

Index Terms - Analog links, dynamic range, low bias, laser intensity noise, microwave photonics, noise reduction, shot noise.

\section{INTRODUCTION}

The use of optical links in analog (RF) signal distribution offers several advantages such as low loss and large bandwidth. In some applications, the links are required to convey signals with a huge span of power levels. Coping with such signals sets stringent requirements for the links' dynamic range (DR), which is defined as the ratio of the largest to the smallest signal power that can be conveyed while preserving certain signal fidelity requirements. This DR is bounded between noise at the lower end and distortion at the upper end. Thus, noise reduction and linearization techniques are of importance in the quest for enhanced DR.

Recently, several papers have been published regarding noise reduction techniques for DR enhancement in analog optical links [1]-[3]. The main idea is to eliminate the (DC) optical carrier power resulting from the pre-biasing of the optical source (this is often dubbed as the low-bias scheme). This carrier power is responsible for the main contribution to the shot noise and relative intensity noise (RIN), which are dominant noise sources in analog optical links. Complete removal of this residual carrier power would result in noise power that scales with the signal power, i.e. weak signals will suffer from lower noise than the strong ones. This feature will lower the minimum detectable signal power and hence, increase the DR.

In this paper, we present simulation results on the performance of a directly-modulated optical link employing a low-bias scheme called Balanced
Modulation and Detection (BMD). In Section II, the principle of $\mathrm{BMD}$ is described. A performance comparison with the so-called Class-AB (CAB) microwave photonic links as described by Darcie et al. [2] is presented in Section III. The links' dynamic range is discussed in Section IV. The paper closes with the conclusion in Section V.

\section{BALANCED MOdULATION AND DETECTION (BMD)}

The schematic of BMD is shown in Fig.1. The modulating RF signal is applied to a pair of laser diodes (LDs) with common input. When the signal is positive, the lower LD will conduct and light is launched in the lower arm of the optical link, while no light is launched in the upper arm. The situation is reversed if the signal is negative. The optical signals in both arms comprise complementary half-wave rectified versions of the original RF signal. Balanced photodetectors (BPDs) are used to restore this signal at the receiving end. Since each arm only carries the half-wave rectified version of the RF signal, both LDs can be biased near the threshold point, which gives virtually zero DC optical power in modern DFB lasers.

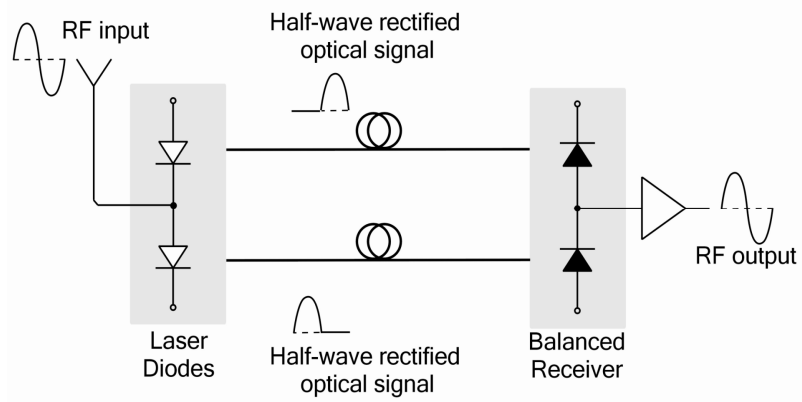

Fig.1. A schematic of a BMD link. The modulating signal is half-wave rectified in the optical domain, allowing the laser diodes to be biased near the threshold.

In links employing BMD, the shot noise in each photodetector is proportional to the mean value of the half-wave rectified signal, while RIN at the output is proportional to the mean squared value of the RF signal. This feature will give huge improvements in links performance compared to the "standard" single-arm intensity modulation-direct detection (IMDD) links 
where the LD is biased roughly half way in the linear portion of the laser characteristic curve. It was shown that for the same signal power, BMD offers an SNR improvement up to $40 \mathrm{~dB}$ compared to the standard IMDD link [1]. In the following section, a model of BMD is developed and calculations of link noise and distortion are performed. The results are compared with a CAB photonic link, which consists of two low-biased Mach-Zehnder modulators (MZMs) combined with a balanced detector.

\section{SiMULATIONS}

\section{A. The BMD Link Model}

The input signal current to the LDs is chosen to be a two-tone sinusoid in the form of

$$
I(t)=I_{\mathrm{m}}\left\{\sin \left(2 \pi f_{1} t\right)+\sin \left(2 \pi f_{2} t\right)\right\},
$$

where $I_{\mathrm{m}}$ is the r.m.s. value of the modulating current, and $f_{1}$ and $f_{2}$ are the modulating frequencies. The current is half-wave rectified and inserted into laser's characteristic containing static nonlinearities up to the third order, which results in optical signal in each arm

$$
P_{n}(t)=c_{1} I_{n}(t)+c_{2} I_{n}^{2}(t)+c_{3} I_{n}^{3}(t),
$$

with $n=\mathrm{x}, \mathrm{y}$ indicates the lower and upper arm, respectively. The expansion coefficients, $c_{1}, c_{2}$ and $c_{3}$ in (2) are chosen to be 0.1 (corresponds to laser slope efficiency), $2.5 \times 10^{-4}$ and $-2.5 \times 10^{-6}$, respectively.

The BPDs are modeled as two photodiodes with responsivities of $R_{\mathrm{x}}$ and $R_{\mathrm{y}}$, which are related to each other by the common-mode rejection ratio (CMRR) defined as

$$
\mathrm{CMRR}=10 \log \frac{\left|R_{\mathrm{x}}-R_{\mathrm{y}}\right|}{R_{\mathrm{x}}+R_{\mathrm{y}}},
$$

with $R_{\mathrm{x}}=R_{\mathrm{y}}=R$ in the case of perfect matching.

Using this model, the detected photocurrent, $I_{\mathrm{D}, \mathrm{BMD}}(t)$, is simply

$$
I_{\mathrm{D}, \mathrm{BMD}}(t)=R_{\mathrm{x}} P_{\mathrm{x}}(t)-R_{\mathrm{y}} P_{\mathrm{y}}(t),
$$

while the mean squared current of the shot noise and RIN are

$$
\sigma_{\text {shot, BMD }}^{2}=2 q B\left\{\left\langle R_{\mathrm{x}} P_{\mathrm{x}}\right\rangle+\left\langle R_{\mathrm{y}} P_{\mathrm{y}}\right\rangle\right\}
$$

and

$$
\sigma_{\mathrm{RIN}, \mathrm{BMD}}^{2}=R I N \cdot B\left\langle I_{\mathrm{D}, \mathrm{BMD}}^{2}(t)\right\rangle,
$$

respectively. In the above equations, $q$ is the electron charge, $B$ single-sided receiver bandwidth, and the operator $\langle\cdot\rangle$ denotes averaging over a period $T$, that is related to the modulating frequencies as $T=1 / \operatorname{gcd}\left(f_{1}, f_{2}\right)$, where gcd is the greatest common divisor.

In order to compute the distortion power, the detected photocurrent, $I_{\mathrm{D}, \mathrm{BMD}}(t)$, is expanded in Fourier series where the basis functions are taken to be $a_{k} \sin (k 2 \pi t / T)$ and $b_{k} \cos (k 2 \pi t / T)$, with $k$ is integer. Thus, the power of a distortion term at frequency $k / T$ is simply $\frac{1}{2}\left(a_{k}^{2}+b_{k}^{2}\right)$.

\section{B. The CAB Link Model}

The model for the CAB link follows directly from [2]. Neglecting the excess loss, the modulated optical power at the MZMs output $\left(P_{+}\right.$and $\left.P_{-}\right)$can be written as

$$
P_{ \pm}=\frac{P_{\mathrm{i}}}{2}\{1-\cos \pi(v \pm \Delta v)\}
$$

where $P_{\mathrm{i}}$ is the input optical power, $v$ and $\Delta v$ are the modulating and bias voltages, respectively, normalized to the switching voltage. At the BPDs, the detected current resulting from the modulated optical power in (7) subtracts, yielding a form of

$$
I_{\mathrm{D}, \mathrm{CAB}}=R P_{\mathrm{i}} \sin (\pi v) \sin (\pi \Delta v),
$$

in case of perfectly matched photodiodes. Just like in the BMD link, the mean squared current of the RIN scales with the detected photocurrent, yielding

$$
\sigma_{\mathrm{RIN}, \mathrm{CAB}}^{2}=R I N \cdot B\left\langle I_{\mathrm{D}, \mathrm{CAB}}{ }^{2}(t)\right\rangle .
$$

However, the mean squared current of the shot noise add from the two photodiodes and thus, depends on the DC optical power in (7), rather than the actual signal power.

$$
\sigma_{\text {shot, } \mathrm{CAB}}^{2}=2 q B R P_{\mathrm{i}}\{1-\cos \pi \Delta v\}
$$

Distortion power in this case is calculated using the same approach as implemented in the case of the BMD link. The normalized modulating voltage is in the form of $\quad v=m\left\{\sin \left(2 \pi f_{1} t\right)+\sin \left(2 \pi f_{2} t\right)\right\}$, with $m$ the modulation index. For the rest of this paper, the normalized bias voltage in the case of the CAB link, $\Delta v$, is set to $1 / 6$, in accordance with [2].

\section{Noise Performance}

To fairly compare the performance of the BMD and the $\mathrm{CAB}$ links, the simulation parameters are chosen such that the detected photocurrents in (4) and (8) yield the same values in the small-signal region. Other common parameters are: thermal noise power spectral density taken to be $-174 \mathrm{dBm} / \mathrm{Hz}, R I N=-145 \mathrm{~dB} / \mathrm{Hz}, P_{\mathrm{i}}$ $=10 \mathrm{~mW}, R=0.8 \mathrm{~A} / \mathrm{W}, f_{1}=750 \mathrm{MHz}, f_{2}=800 \mathrm{MHz}$ and the load resistance, $R_{\mathrm{L}}$, equals to $50 \Omega$.

The plot of noise power for the BMD and the CAB links are shown in Fig. 2 and Fig. 3, respectively. In the former case, both RIN and shot noise scale with the 
signal power. The link is thermal noise-limited for low RF power and directly shifts to RIN-limited in the high RF power region without being shot noise-limited. This is due to the fact that shot noise is largely reduced below the other two noise sources.

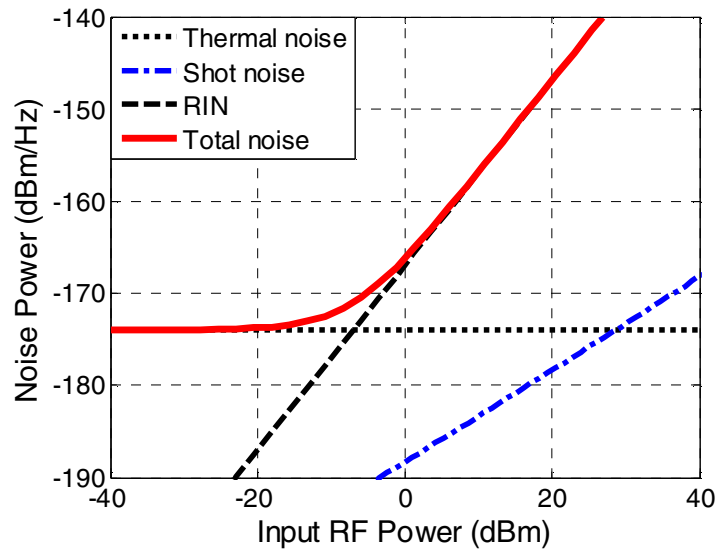

Fig. 2. Noise power in $1 \mathrm{~Hz}$ bandwidth for the BMD link. Both shot noise and RIN scale with the RF signal power. The link is limited by thermal noise for low RF power and RIN limited for high RF power. Noise reduction in this link is insensitive to CMRR of the BPDs.

In the CAB link, the RIN scales with the signal power but the shot noise doesn't. This will result in a link that is shot noise-limited in the low RF power region and RINlimited for high RF power. Unlike in the BMD link, the RIN reduction in the $\mathrm{CAB}$ link is sensitive to the imbalance that might occur in the BPDs.

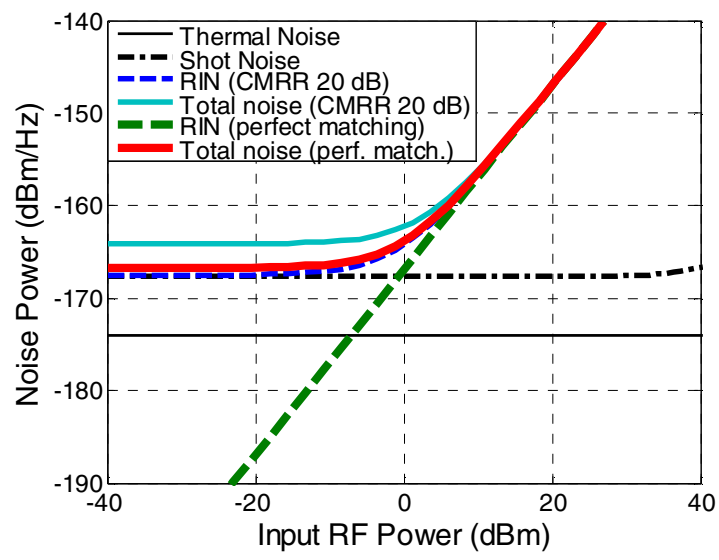

Fig. 3. Noise power in $1 \mathrm{~Hz}$ bandwidth for the CAB link. For a perfectly matched link, RIN scales with RF signal power whereas shot noise remains constant. RIN reduction in this link is sensitive to the CMRR of the BPDs.

In Fig. 4, the signal-to-noise ratio (SNR) of the two links in a $1 \mathrm{GHz}$ bandwidth is depicted. The BMD link benefits an improvement around 7.2 dB in SNR over the $\mathrm{CAB}$ link for low signal power. If there is a mismatch in the BPDs (for example $\mathrm{CMRR}=20 \mathrm{~dB}$ ), this improvement in SNR rises to $8.7 \mathrm{~dB}$. For high signal power, both links are RIN limited and they yield the same noise performance.

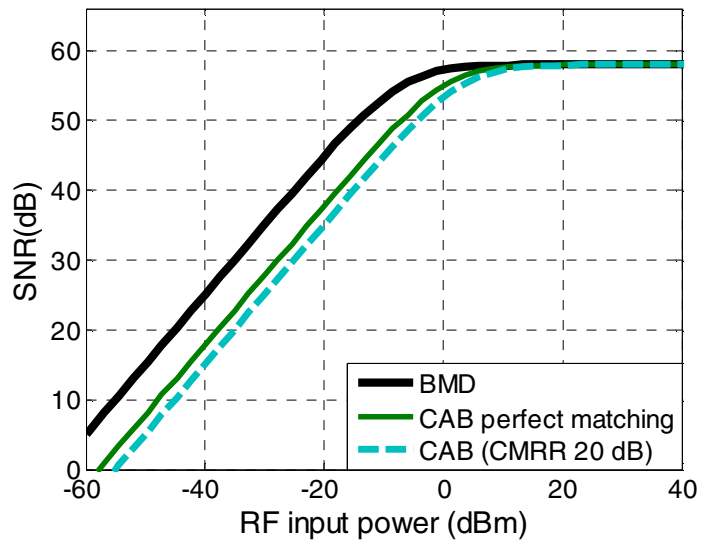

Fig. 4. SNR of the BMD and the CAB links. The BMD link has an advantage of SNR ranging from 7.2 to $8.7 \mathrm{~dB}$ over the CAB link depending on CMRR of the BPDs.

\section{Distortion}

Due to the rectification of the input RF signal, the BMD link yields higher distortions compared to the $\mathrm{CAB}$ link. Even though both links benefit from second-order distortion suppression, this feature is highly sensitive to the matching of the BPDs. This is especially true for the BMD link, as depicted in Fig. 5.

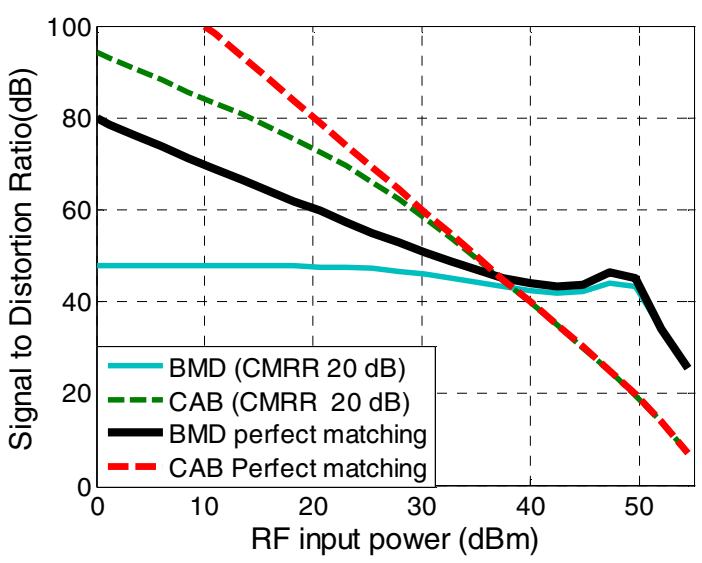

Fig. 5. Signal-to-distortion ratio (SDR) for the BMD and the CAB links. All distortion terms within a $1 \mathrm{GHz}$ bandwidth, from $570 \mathrm{MHz}$ to $1.57 \mathrm{GHz}$ are included.

In the above figure, signal-to-distortion ratio (SDR) is calculated in a $1 \mathrm{GHz}$ bandwidth incorporating all distortion terms. Since the frequency range is chosen to run from $570 \mathrm{MHz}$ to $1.57 \mathrm{GHz}$, second-order harmonic (H2) and intermodulation (IM2) will fall inside the band of interest. The reason to choose this frequency range is to cope with applications in which H2 and IM2 cannot be filtered out. Based on the calculation results developed in this section, the resulting DR of both links will be presented and discussed in the next section. 


\section{DISCUSSION}

\section{A. Dynamic Range}

The most common definition of DR is the difference (in $\mathrm{dB}$ ) between the highest output RF power that meets $0 \mathrm{~dB}$ distortion-to-noise ratio and the lowest output $\mathrm{RF}$ that meets $0 \mathrm{~dB}$ SNR, referenced to a $1 \mathrm{~Hz}$ bandwidth [4]. This definition is known as the spurious-free dynamic range (SFDR). However, this definition is not suitable to use in the situation where the noise floor is not constant but depends on the input RF power. Moreover, in actual applications, certain values of minimum SNR and minimum SDR are prescribed, rather than $0 \mathrm{~dB}$ of SNR and distortion-to-noise ratio, making the SFDR definition useless for practical applications. Thus, it is more useful to define DR as the range of input RF power that fulfills a minimum required SNR and at the same time fulfills a specified minimum SDR in a given signal bandwidth. This definition is preferred over the SFDR definition especially in the field of radio astronomy [5].

Using the above mentioned definition and setting the minimum SNR and the minimum SDR in a $1 \mathrm{GHz}$ bandwidth as $20 \mathrm{~dB}$ and $40 \mathrm{~dB}$ respectively [5], the DR can be roughly determined from Fig. 4 and Fig. 5. With a more precise calculation, the $\mathrm{DR}$ for the BMD link is found to be $95.7 \mathrm{~dB}$, which is an $18 \mathrm{~dB}$ advantage compared to the $\mathrm{CAB}$ link with $\mathrm{DR}$ of $77.7 \mathrm{~dB}$.

A more general picture of the achievable DR can be obtained by considering Fig. 6. Here, the achievable DR for both links is depicted as a function of the required minimum SDR, using a practical CMRR value of $30 \mathrm{~dB}$ and a fixed minimum required SNR of $20 \mathrm{~dB}$. For minimum SDR values up to $55 \mathrm{~dB}$, the BMD link still outperforms the $\mathrm{CAB}$ link. Beyond this value, the achievable DR of the BMD link deteriorates faster than that of the CAB link. However, a better performance of BMD can be expected if LDs with better linearity are employed.

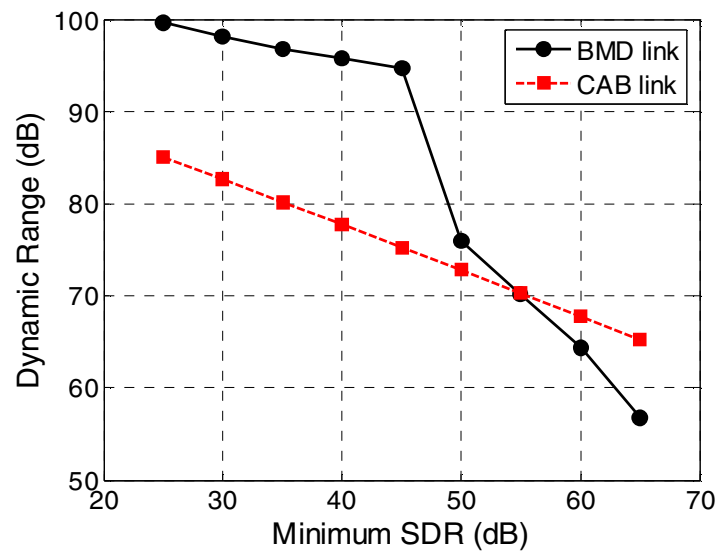

Fig. 6. The achievable DR for both BMD and CAB links as a function of minimum SDR prescribed by the applications. The CMRR is taken to be $30 \mathrm{~dB}$ and the minimum required SNR is fixed at $20 \mathrm{~dB}$.

\section{B. Potential Applications}

Unlike the other noise reduction schemes, for example the $\mathrm{CAB}$ link and the so-called the class-B photonic link [3], which require external modulators, the BMD scheme can be implemented in directly-modulated links. This feature is attractive when complexity and cost considerations come into the picture. In applications like phased-array antenna receivers for radio astronomy, the number of optical links required might be very large. Hence, employing links with external modulators might become too costly. With the BMD scheme, enhanced DR is achievable in simple and possibly low cost links.

\section{CONCLUSION}

A modulation scheme that reduces the noise and enhances the dynamic range of a directly-modulated optical link has been presented. Rectification of the modulating RF signal resulted in noise powers that scales with the input signal power, hence, enhance the links performance. A comparison with the CAB link has been made and an advantage up to $8.7 \mathrm{~dB}$ in the link SNR and $18 \mathrm{~dB}$ in dynamic range are achieved.

\section{ACKNOWLEDGEMENT}

This work is a part of the PACMAN project which is supported by the Dutch Ministry of Economic Affairs, SenterNovem project number TSIT3049.

\section{REFERENCES}

[1] D.A.I. Marpaung, C.G.H. Roeloffzen, W. van Etten, "A novel modulation scheme for noise reduction in analog fiber-optic links", Proc. of the $9^{\text {th }}$ IEEE/LEOS Symp. in the Benelux, Mons, Belgium, December 2005, pp. 113-116.

[2] T.E. Darcie, A. Moye, P.F. Driessen, J.D. Bull, H. Kato, N.A.F. Jaeger, "Noise reduction in class-AB microwave-photonic links", IEEE MWP 2005, Seoul, South Korea, October 12-14, 2005, pp. 329332.

[3] T.E. Darcie, J. Zhang, P.F. Driessen, J.J. Eun, "Demonstration of a class-B microwave-photonic link using optical frequency modulation and complementary fiber-Bragg-grating discriminators", OFC/NFOEC 2006, Anaheim, CA, March 6-10, 2006, Paper PDP38.

[4] C. H. Cox, III, Analog Optical Links : Theory and Practice, Cambridge, 2004.

[5] B. Smith, R.E. Spencer, D.C. Brown, M. Bentley, "Optical fibre communications between radio telescopes in the European VLBI network", TMRLSF RTD Sub Project 4, 2000. 